\title{
Investigation of muscle-specific beef color stability at different ultimate $\mathrm{pHs}$
}

\author{
Shuang Wu ${ }^{1}$, Jina Han ${ }^{1}$, Rongrong Liang ${ }^{1}$, Pengcheng Dong ${ }^{1}$, Lixian $\mathrm{Zhu}^{1}$, \\ David L. Hopkins ${ }^{1,2}$, Yimin Zhang ${ }^{1, *}$, and Xin Luo ${ }^{1, *}$
}

\author{
* Corresponding Authors: \\ Yimin Zhang \\ Tel: +86-538-8242745, Fax: +86-538-8242745, \\ E-mail:ymzhang@sdau.edu.cn \\ Xin Luo \\ Tel: +86-538-8248255, Fax: +86-538-8242745, \\ E-mail: luoxin@sdau.edu.cn
}

'Lab of Beef Processing and Quality Control, College of Food Science and Engineering, Shandong Agricultural University, Taian, Shandong 271018, China

${ }^{2}$ NSW Department of Primary Industries, Centre for Red Meat and Sheep Development, PO Box 129, Cowra NSW 2794, Australia

ORCID

Shuang Wu

https://orcid.org/0000-0001-6801-0000

Jina Han

https://orcid.org/0000-0001-6301-5814

Rongrong Liang

https://orcid. org/0000-0002-8536-1520

Pengcheng Dong

https://orcid.org/0000-0001-8994-2608

Lixian Zhu

https://orcid.org/0000-0002-1252-4720

David L. Hopkins

https://orcid.org/0000-0002-2743-5894

Yimin Zhang

https://orcid.org/0000-0001-5240-7126

Xin Luo

https://orcid.org/0000-0002-8583-8093

Submitted Dec 10, 2019; Revised Jan 7, 2020; Accepted Jan 28, 2020
Objective: This study was aimed to investigate the muscle-specific beef color stability at normal and high ultimate $\mathrm{pHs}$.

Methods: The impact of muscle (Longissimus lumborum [LL] vs psoas major [PM]) and $\mathrm{pH}$ (normal ultimate $\mathrm{pH}[\mathrm{Np}]$ vs high $\mathrm{pH}$ dark cutting beef [Hp]) on color stability, indicated by basic color traits, metmyoglobin reducing activity (MRA) and oxygen consumption (OC), as well as the lipid oxidation, were determined over 7 days of display at $4^{\circ} \mathrm{C}$.

Results: Hp-LL had the highest $\mathrm{pH}$ (6.92), followed by Hp-PM (6.01), Np-PM (5.76), and Np-LL (5.52). Hp-LL had increased $(\mathrm{p}<0.05) a^{*}$, chroma and \% oxymyoglobin during display. Hp-LL also had the highest metmyoglobin $(\mathrm{MMb})$ reducing activity and $\mathrm{OC}$ among all the samples, thus, the greatest color stability, although very dark throughout storage, with lowest values for lightness $\left(L^{*}\right)$ and yellowness $\left(b^{*}\right)$. Np-LL also exhibited relatively high color stability, as a result of its lower \% MMb and OC and higher MRA than psoas muscle samples. The 0.2 unit difference of the $\mathrm{pH}$ between $\mathrm{Hp}$ and $\mathrm{Np}$ psoas muscle, resulted in the difference of the color intensity, not the color stability. Interestingly, high $\mathrm{pH}$ psoas muscle (Hp-PM) did not have better color stability than Np-PM, and in fact had lower color stability than even Np-LL. The similar level of OC and lipid oxidation cannot explain the difference in color stability between Hp-PM and Np-LL.

Conclusion: The Hp does not always show better color stability compared with Np beef, which depends on the muscle type. The balance of MRA and OC is important to keep the color in great intensity and stability in the meantime.

Keywords: Beef Color; Dark Cutting; Oxygen Consumption; Metmyoglobin Reducing Activity; Ultimate $\mathrm{pH}$

\section{INTRODUCTION}

Meat color is one of the most important quality traits influencing consumer purchasing decisions, since consumers generally evaluate freshness and wholesomeness of meat through surface color [1]. Fresh beef color is mainly determined by myoglobin content and its redox state. Deoxymyoglobin (DMb) exists as a purplish-red color usually occurring on fresh cut surfaces, and it converts to a bright cherry red when exposed to air or oxygen (oxymyoglobin $[\mathrm{OMb}])$. However, metmyoglobin $(\mathrm{MMb})$ can lead to a brown color through the oxidation of ferrous myoglobin [2]. Generally, dark cutting beef is rejected by most consumers due to appearance. In fact, brownish discoloration or dark color caused a loss of $\$ 1$ billion and $\$ 165$ to $\$ 170$ million, respectively, to the US beef industry in $2000[3,4]$.

Of several endogenous factors influencing beef color and color stability, muscle type and ultimate $\mathrm{pH}$ have received significant attention. Several previous studies have reported that meat color stability is a muscle-specific trait, due to the variable proportion of metabolic 
fiber types (oxidative or glycolytic) among muscles $[5,6]$. Compared with color-stable Longissimus lumborum (LL), biochemical studies have documented that psoas major (PM) is a color-labile muscle $[7,8]$. Dark cutting beef is associated with a high ultimate $\mathrm{pH}(\mathrm{Hp})$, and the increased ultimate $\mathrm{pH}$ enhances water-holding capacity and mitochondrial activity, which lead to less light scattering and the formation of more $\mathrm{DMb}$ than normal $\mathrm{pH}(\mathrm{Np})$ beef, resulting in a darker surface color [9-11]. Interestingly, although Hp has less redness intensity, it demonstrates greater oxygen consumption (OC), and notably greater color stability than $\mathrm{Np}$ beef $[12,13]$.

Several research groups have investigated the color values of different muscles from Hp beef. Some study reported that dark cutting status had various effects on color $L^{\star}, a^{\star}, b^{*}$ values of hindquarter muscles at 7 days postmortem [14]. Holman and Hopkins [15] compared the eating quality traits of aged bolar blade, striploin and topside cuts of $\mathrm{Hp}$ beef, and found that the largest dark cutting effect was in the striploin. However, they did not compare the interaction effects of cut (muscle type) and ultimate $\mathrm{pH}$ on color traits and color stability, and especially, the underlying mechanisms of those color differences.

Therefore, in this study, the color stability comparison of LL and PM from Np and Hp beef, under aerobic packaging during retail display was studied. This was to ascertain the order of color stability between different muscles, which will provide more information on the underlying mechanisms of color stability as affected by muscle type and $\mathrm{pHu}$.

\section{MATERIALS AND METHODS}

\section{Animal and sample preparation}

Beef carcasses (24 months old, Luxi Yellow $\times$ Simmental cattle, 300 to $350 \mathrm{~kg}$ carcass weight) were randomly selected from a commercial abattoir. Fourteen carcasses were assigned to 2 groups based on observed muscle $\mathrm{pH}$ at approximately $24 \mathrm{~h}$ postmortem: Hp beef (also called dark, firm and dry beef, which exhibits a significantly darker color and higher $\mathrm{pH}$ value compared with normal beef. In China, the threshold of $\mathrm{Hp}$ beef was defined as 6.1 [16]) group $(\mathrm{pH}>6.1, \mathrm{n}=7)$ and $\mathrm{Np}$ value group (pH 5.4 to $5.8, \mathrm{n}=7$ ). Muscle $\mathrm{pH}$ was collected on the anterior surface of LL between the 12-13th rib on the right side of each carcass using a portable $\mathrm{pH}$ meter (Senven2Go-S2, Mettler-Toledo, Greifensee, Switzerland), which was calibrated with two buffers ( $\mathrm{pH} 4.0$ and 7.0); each carcass was measured twice. LL and PM were removed from right carcass sides at $48 \mathrm{~h}$ postmortem (chilling at $0^{\circ} \mathrm{C}$ to $4^{\circ} \mathrm{C}$ ), commercially vacuum packaged, and then transported to the laboratory on ice.

Following overnight storage, LL and PM from the same carcass were fabricated into ten 2.5 - $\mathrm{cm}$-thick steaks, and eight steaks were randomly assigned to day $0,3,5$, and 7 for retail display (two steaks on each day as duplicates). The remaining 2 steaks were randomly assigned to day 0 and 7 for measuring total viable microbial count. Steaks were placed on foam trays with absorbent pads (DLS-25, Sealed Air Corp., Danbury, CT, USA), overwrapped with Polyethylene (PE) film (water vapor permeability: $23.5 \mathrm{~g} / \mathrm{m}^{2} / 24 \mathrm{~h}$, oxygen transmission rate: $16,654 \mathrm{~cm}^{3} / \mathrm{m}^{2} / 24 \mathrm{~h} / \mathrm{atm}$ ), and stored in a walk-in cooler at $2^{\circ} \mathrm{C} \pm 1^{\circ} \mathrm{C}$ under continuous lighting (1,600 to 2,000 lx, Leishi Warm Yellow Light-Emitting Diode Light; color temperature $=3,000 \mathrm{~K}$ ). All steaks were rotated daily to minimize the variance in light intensity or temperature caused by the location. The following traits were measured at each time point: $\mathrm{pH}$, surface color traits $\left(L^{*}, a^{*}, b^{\star}\right.$, Chroma and hue values, R630/580, relative myoglobin content), OC, and metmyoglobin reducing activity (MRA). Samples for subsequent lipid oxidation analysis were obtained at each sampling timepoint and stored at $-80^{\circ} \mathrm{C}$.

\section{Proximate components and $\mathrm{pH}$}

Moisture, protein and fat content of the steaks was determined based on National Standards of the People's Republic of China (GB/T 9695.15-2008, GB/T 5009.5-2010, and GB/T 9695.152008 , respectively), and the results were reported on a percent (\%) basis. $\mathrm{pH}$ value of each steak was measured directly using a portable $\mathrm{pH}$ meter, with the meter calibrated using two buffers ( $\mathrm{pH} 4.00$ and 7.00$)$ at $4^{\circ} \mathrm{C}$. The probe was inserted into each steak (about $2 \mathrm{~cm}$ depth) at four different locations, and $\mathrm{pH}$ values were averaged.

\section{Total viable counts}

Steak surface samples ( $3 \mathrm{~mm}$ thick slices; $10 \mathrm{~g}$ ) were taken aseptically, chopped, and transferred to sterile lateral filter bags (BagPage; Interscience, St Nom, France), with addition of $90 \mathrm{~mL}$ of sterile tryptone salt solution at $0.85 \%$ (w/v). Samples were mixed with a blender (BagMixer 400; Interscience, France) for $2 \mathrm{~min}$ at room temperature. A 10 -fold dilution series was prepared to perform microbial analysis. Diluted sample solutions were cultured on plate count agar (LandBridge Co., Ltd., Beijing, China) and incubated at $37^{\circ} \mathrm{C}$ for $48 \mathrm{~h}$. Results were expressed as log colony-forming unit (CFU)/ g sample.

\section{Color attributes}

The surface color of steaks on display day 0 (blooming for 1 h at $0^{\circ} \mathrm{C}$ to $\left.4^{\circ} \mathrm{C}\right)$, days 3,5 , and 7 were measured by a colorimeter (Model SP62; $8 \mathrm{~mm}$ diameter aperture, Illuminant A, $10^{\circ}$ observer; X-Rite, Inc., Grand Rapids, MI, USA). The Commission Internationale de l'Eclairage $L^{*}$ (lightness), $a^{*}$ (redness), and $b^{*}$ (yellowness) values of each steak were measured four times and averaged. Chroma and hue values were calculated using the equation: $\left(\left[a^{*^{2}}+b^{*^{2}}\right]^{0.5}\right)$ and (arctangent 
$\left.\left[b^{*} / a^{*}\right]\right)$, respectively. The reflected wavelengths of the instrument were recorded in the range of 400 to $700 \mathrm{~nm}$ at 10 $\mathrm{nm}$ intervals and the ratio of reflectance at $630 \mathrm{~nm}$ and 580 $\mathrm{nm}$ (R630/580) was used to directly evaluate the color stability during display. The reflectance $(\mathrm{R})$ at $473,525,572$, and 700 $\mathrm{nm}$ were converted to reflex attenuance $(\mathrm{A})$ using the equation: $A=\log (1 / R)$ and the relative percentage of three myoglobin redox forms were calculated following the equations as described by AMSA [17]:

$$
\begin{aligned}
& \% \mathrm{MMb}=\left(1.395-\frac{A 572-A 700}{A 525-A 700}\right) \times 100 \\
& \% \mathrm{DMb}=\left[2.35 \times\left(1-\frac{A 473-\mathrm{A} 700}{A 525-A 700}\right)\right] \times 100 \\
& \% \mathrm{OMb}=100-(\% \mathrm{MMb}+\% \mathrm{DMb})
\end{aligned}
$$

\section{Metmyoglobin reducing activity and oxygen consumption}

Several studies have reported that the initial MMb formed is a good indicator of muscle MRA $[5,18]$. Thus, MRA was measured as resistance to nitrite induced myoglobin oxidation to MMb. MRA determination was conducted according to the method described by Sammel et al [19] and Mancini et al [18], for which a lower initial $\mathrm{MMb}$ formation value indicates greater MRA. A cube $\left(2.54 \times 2.54 \times 2.54 \mathrm{~cm}^{3}\right)$ with no connective tissue or visible fat was removed from the central location of each steak. Then each cube was bisected horizontally, resulting in two half pieces. The top piece (including the surface exposed to light) was submerged in a $0.3 \%(\mathrm{w} / \mathrm{v})$ solution of sodium nitrite for $20 \mathrm{~min}$ at $20^{\circ} \mathrm{C}$, then was removed, blotted dry, and the reflectance of sample surface immediately measured by the colorimeter mentioned above to determine the initial $\mathrm{MMb}$ formation. The initial MMb formation was calculated by the formula:

$\% \mathrm{MMb}$

$$
=\frac{\frac{\mathrm{K} / \mathrm{S} 572}{\mathrm{~K} / \mathrm{S} 525} \text { for } 100 \% \mathrm{DMb}-\frac{\mathrm{K} / \mathrm{S} 572}{\mathrm{~K} / \mathrm{S} 525} \text { for sample }}{\frac{\mathrm{K} / \mathrm{S} 572}{\mathrm{~K} / \mathrm{S} 525} \text { for } 100 \% \mathrm{DMb}-\frac{\mathrm{K} / \mathrm{S} 572}{\mathrm{~K} / \mathrm{S} 525} \text { for } 100 \% \mathrm{MMb}}
$$

(AMSA [17]).

The $100 \% \mathrm{DMb}$ and $100 \% \mathrm{MMb}$ were measured according to section IX B2a and section IX B1a of AMSA [17], respectively. Samples of day 0 was used to measure the $100 \% \mathrm{DMb}$ and samples of day 3 for $100 \% \mathrm{MMb}$ to format the most complete $\mathrm{MMb}$.

Muscle OC was measured as described by Madhavi and Carpenter [20] with a modification. The freshly cut surface of the bottom half was covered with $\mathrm{PE}$ film as previously mentioned and bloomed for $2 \mathrm{~h}$ at $2^{\circ} \mathrm{C}$, vacuum packaged, and immediately the reflectance of the bloomed surface was measured to determine initial $\mathrm{OMb}$ content by using $\mathrm{K} / \mathrm{S}$ ra- tios and equations [17]. The packaged sample was incubated at $30^{\circ} \mathrm{C}$ for $30 \mathrm{~min}$ and then measured again to determine the final $\mathrm{OMb}$ content. The $\mathrm{OC}$ was calculated following the equation:

$$
\text { [(Initial OMb \% - final OMb \%)/initial OMb \%]×100 }
$$

\section{Lipid oxidation}

Lipid oxidation was evaluated through 2-thiobarbituric acid reactive substances values according to a modified procedure of Siu and Draper [21]. Four gram of sample was homogenized for $1 \mathrm{~min}$ in $20 \mathrm{~mL}$ of distilled water by an Ultra-Turrax T18 homogenizer (T18; IKA, Staufen, Germany). Subsequently, $20 \mathrm{~mL}$ of $10 \%(\mathrm{w} / \mathrm{v})$ trichloroacetic acid was added into the homogenate and vortex-blended, then filtered through Whatman (\#1) filter paper. Amount of $1 \mathrm{~mL}$ of $60 \mathrm{mM} 2$-thiobarbituric acid solution was mixed with $4 \mathrm{~mL}$ of filtrate and incubated in a water bath at $80^{\circ} \mathrm{C}$ for $90 \mathrm{~min}$. After the solution was cooled to room temperature, absorbance was measured using a microplate spectrophotometer (Epoch 2, Bio Tek Instruments, Winooski, VT, USA) at $532 \mathrm{~nm}$ and calculated with a standard curve of 1,1,3,3,-Tetraethoxypropane solution (Sigma, St. Louis, MO, USA). The results were expressed as 2-thiobarbituric acid reactive substances in $\mathrm{mg}$ malondialdehhyde/kg meat.

\section{Statistical analysis}

In this experiment, statistical analysis was performed using a MIXED procedure (SAS, Version 9.0, Cary, NC, USA). Muscle type (LL and PM), ultimate $\mathrm{pH}(\mathrm{Np}$ and $\mathrm{Hp}$ ), display time and their interactions were fixed factors and carcass was a random factoProximate components for the analysis for the $\mathrm{pH}$, total viable counts, color attributes, relative content of myoglobin, MRA, OC and lipid oxidation. While muscle type ( $\mathrm{LL}$ and $\mathrm{PM}$ ), ultimate $\mathrm{pH}$ ( $\mathrm{Np}$ and $\mathrm{Hp}$ ) and their interaction were considered as fixed factors and carcass as a random factor. Tests of differences between predicted means were applied using the PDIFF statement and differences were considered significantly different at $\mathrm{p}<0.05$.

\section{RESULTS AND DISCUSSION}

\section{$\mathrm{pH}$, proximate composition and total viable counts}

$p H$ values: No interaction effect ( $\mathrm{p}>0.05$ ) of muscle type, ultimate $\mathrm{pH}(\mathrm{pHu})$ and display time was found for $\mathrm{pH}$ values, but there was an interaction $(\mathrm{p}<0.05)$ effect of $\mathrm{pHu} \times$ muscle type on $\mathrm{pH}$ values $(\mathrm{p}<0.05)$ (Table 1$)$, with much smaller differences within the PM muscle for the two $\mathrm{pH}$ groups. For both LL and PM muscles, $\mathrm{Hp}$ beef had a greater $\mathrm{pH}$ than $\mathrm{Np}$ beef $(\mathrm{p}<0.05)$, and the LL exhibited lower $(\mathrm{p}<0.05) \mathrm{pH}$ values than PM from Np beef (Table 1). However, among Hp samples, LL had higher $\mathrm{pH}$ values ( $\mathrm{p}<0.05$; Table 1$)$ than PM. 
Table 1. Interaction effects of muscle type and ultimate $\mathrm{pH}$ on $\mathrm{pH}$ and proximate composition

\begin{tabular}{lcccccr}
\hline \multirow{2}{*}{ Trait } & \multicolumn{2}{c}{ LL } & & \multicolumn{2}{c}{ PM } & \multirow{2}{*}{ SE } \\
\cline { 2 - 3 } & Normal pH & High pH & & Normal pH & High pH & \\
\hline pH & $5.52^{\mathrm{d}}$ & $6.93^{\mathrm{a}}$ & & $5.77^{\mathrm{c}}$ & $5.96^{\mathrm{b}}$ & 0.05 \\
Moisture (\%) & $73.81^{\mathrm{b}}$ & $75.98^{\mathrm{a}}$ & & $74.45^{\mathrm{ab}}$ & $75.27^{\mathrm{a}}$ & 0.60 \\
Protein (\%) & 22.63 & 22.98 & & 23.26 & 22.72 & 0.28 \\
Fat (\%) & $1.96^{\mathrm{a}}$ & $0.84^{\mathrm{b}}$ & & $1.66^{\mathrm{a}}$ & $1.44^{\mathrm{a}}$ & 0.21 \\
\hline
\end{tabular}

$\mathrm{LL}$, Iongissimus lumborum; PM, psoas major; SE, standard error.

${ }^{a-d}$ Means in the columns with different letters differ at $p<0.05$.

The higher $\mathrm{pH}$ values in the PM than those in the LL from Np beef have been reported previously [7,22]. Lefaucheur [23] reported that glycolytic muscle (such as LL) exhibited higher glycogen levels and higher actomyosin-ATPase activity compared to oxidative muscle (such as PM), which can stimulate post-mortem glycolysis and increase the accumulation of lactic acid, producing a greater decline in $\mathrm{pH}$. However, more recent work has reported that oxidative muscle produces meat with a high $\mathrm{pHu}$ regardless of glycogen content [24]. Furthermore, PM possesses more slow-switch type I fibres, and the proportion of type I fibres is negatively correlated with $\mathrm{pH}$ and glycolytic indicators at $24 \mathrm{~h}$ postmortem [25]. Thus, the different muscle fibre composition between $\mathrm{LL}$ and $\mathrm{PM}$ of $\mathrm{Np}$ beef plays a decisive role in muscle $\mathrm{pH}$ as previously reported.

It is interesting to find that the $\mathrm{pH}$ of $\mathrm{PM}$ was lower than that of LL from Hp beef. Wulf et al [14] also reported a $\mathrm{pH}$ difference between LL and PM muscles of Hp (6.00 vs 5.80), but the $\mathrm{pH}$ of the tensor fasciae latae and rectus femoris from Hp beef were not different (5.66 vs 5.67). Recently, Holman and Hopkins [15] reported that the dark cutting effect on the $\mathrm{pH}$ of different cuts (muscles) was not uniform. However, the $\mathrm{pH}$ of $\mathrm{PM}$ was higher than $\mathrm{LL}$, while the value was lower than LL from Hp beef. This inconsistency needs further exploration, possibly due to differences in glycogen content or glycogen consumption rate between muscle types or $\mathrm{pHu}$ groups.

Proximate composition: There was no interaction effect ( $\mathrm{p}>0.05$ ) between muscle type and $\mathrm{pHu}$ on proximate com- position. While $\mathrm{pHu}$ had a significant effect on moisture and fat content, neither $\mathrm{pHu}$ nor muscle type had an effect $(\mathrm{p}>0.05)$ on protein content (Table 1). Hp muscle showed greater moisture $(\mathrm{p}<0.05)$ and lower fat content $(\mathrm{p}<0.05)$ than Np muscle, which is consistent with the results of English et al [12]. The higher water content in $\mathrm{Hp}$ beef, may be due to depletion of fat depots via increased fatty acid oxidation in $\mathrm{Hp}$ [26]. Also, the higher $\mathrm{pHu}$ of $\mathrm{Hp}$ muscle enhances water holding capacity, as this moves the major proteins in muscle away from the isoelectric point ( $\mathrm{pH} 5.0$ to 5.5), causing the proteins to have a negative charge which increases their ability to bind water. Sawyer et al [27] also documented that Hp beef showed greater bound water and lower free water levels than $\mathrm{Np}$ beef.

Total viable count: A significant $\mathrm{pHu} \times$ muscle type $\times$ display time interaction $(\mathrm{p}<0.05)$ on total viable count (TVC) was found (Table 2). The initial bacterial counts for the $\mathrm{Np}$ LL were higher than other samples $(\mathrm{p}<0.05)$. As display time extended to $7 \mathrm{~d}$, TVC increased $(\mathrm{p}<0.05)$ significantly for all muscles. However, for both LL and PM, TVC in the Hp group were higher than the $\mathrm{Np}$ group on display day 7, reaching 6.61 to $6.37 \log$ CFU/g. This finding is in agreement with previous studies, which reported that muscle with high $\mathrm{pH}$ was more prone to spoilage, reflecting low or absent glycogen levels of high $\mathrm{pH}$ muscle [10]. This is a major issue for the utilization of $\mathrm{Hp}$ beef.

\section{Color attributes}

$L^{*}$ values: There was a significant $\mathrm{pHu} \times$ muscle type interaction $(\mathrm{p}<0.05)$ for $L^{*}$ values (Table 3$) . L^{*}$ values of Hp-LL were the lowest $(p<0.05)$ among all samples, followed by Hp$\mathrm{PM}$, and there was no difference in $L^{*}$ values between $\mathrm{Np}$ LL and Np-PM. In support, several previous studies have shown that $\mathrm{Hp}$ beef had lower $L^{*}$ values than $\mathrm{Np}$ beef, and LL from Np beef exhibited higher $L^{*}$ values than PM [14,2729]. The differences in $L^{*}$ values between $\mathrm{Np}$ and $\mathrm{Hp}$ beef can be attributed to the $\mathrm{pHu}$ values. As muscle $\mathrm{pH}$ values increase, the water holding capacity of proteins increases, and the higher intracellular water content can cause swelling of fibers and shrinkage of the space between muscle fibrils. This decreases light scattering and increases light absorption by

Table 2. Effects of muscle type, ultimate pH and display time on total viable count

\begin{tabular}{|c|c|c|c|c|c|}
\hline \multirow{2}{*}{ Trait } & \multirow{2}{*}{ Muscle type } & \multirow{2}{*}{ Ultimate $\mathrm{pH}$} & \multicolumn{2}{|c|}{ Display time (d) } & \multirow{2}{*}{ SE } \\
\hline & & & 0 & 7 & \\
\hline \multirow[t]{4}{*}{ TVC $(\log$ CFU/g) } & $\mathrm{LL}$ & Normal pH & $2.90^{\mathrm{aw}}$ & $3.88^{\text {by }}$ & 0.02 \\
\hline & & High pH & $2.13^{\mathrm{ax}}$ & $6.61^{\mathrm{bw}}$ & \\
\hline & PM & Normal pH & $2.48^{\operatorname{anx} x}$ & $4.86^{b x}$ & \\
\hline & & High pH & $2.30^{a x}$ & $6.37^{\mathrm{bw}}$ & \\
\hline
\end{tabular}

SE, standard error; TVC, total viable count; CFU, colony-forming unit; LL, longissimus lumborum; PM, psoas major.

$a, b$ Means in the rows with different letters differ at $p<0.05$.

${ }^{w-y}$ Means in the columns with different letters differ at $p<0.05$. 
Table 3. Effects of muscle type, ultimate $\mathrm{pH}$ and display time on meat color

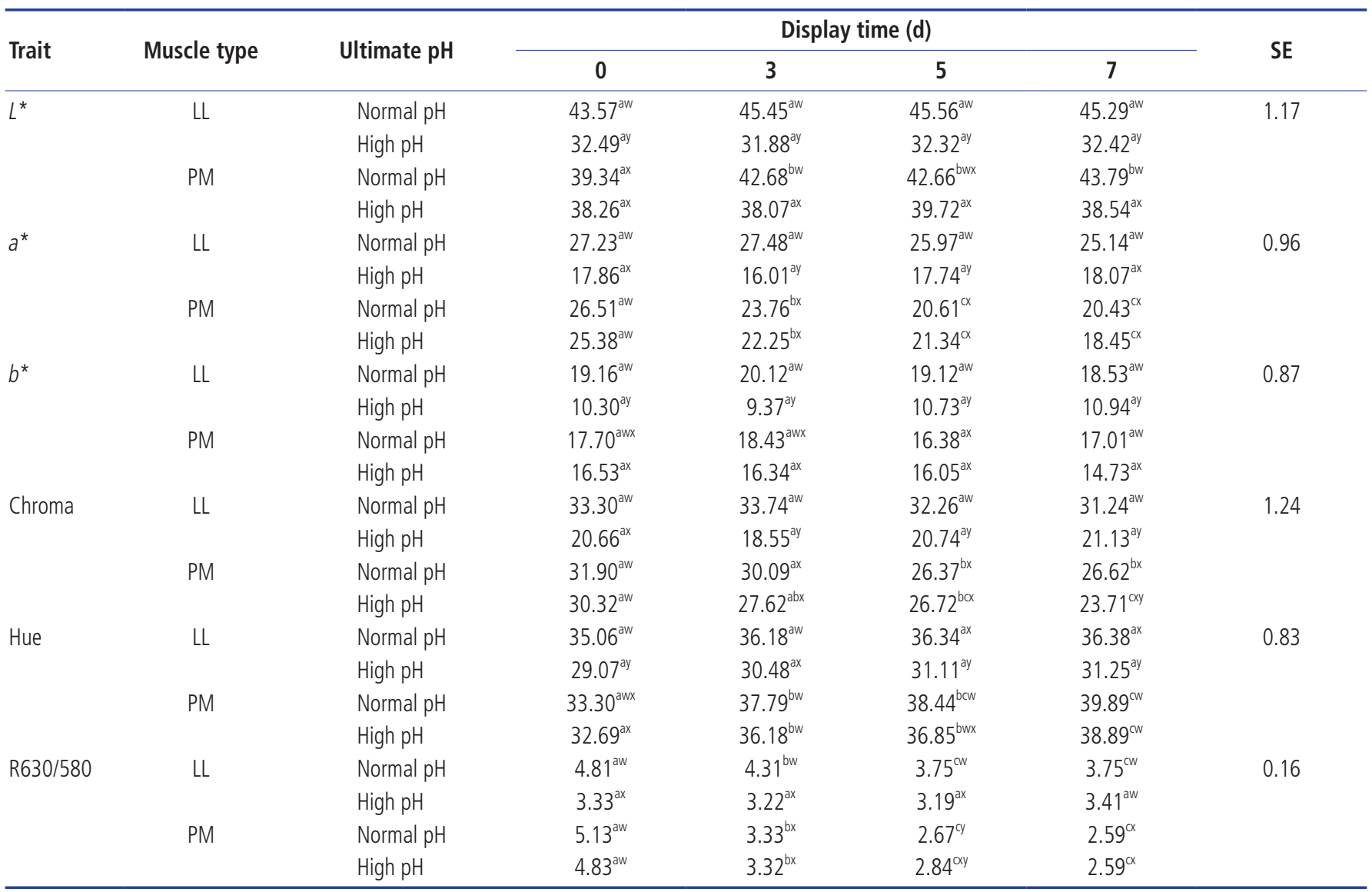

SE, standard error; LL, longissimus lumborum; PM, psoas major.

${ }^{a-c}$ Means in the rows with different letters differ at $p<0.05$.

${ }^{w-y}$ Means in the columns within a trait with different letters differ at $p<0.05$.

myoglobin. Consequently, the muscle surface color appears darker [11].

$a^{*}, b^{*}$, and chroma values: Significant interactions of muscle type $\times \mathrm{pHu}$ and mscle type $\times$ display time occurred for $a^{\star}, b^{\star}$, chroma and hue values (Table 2). During display, all samples, except Hp-LL, showed decreased $(\mathrm{p}<0.05) a^{*}$ values, and the decrease was greater in PM from both $\mathrm{pHu}$ beef than that in Np-LL, indicating a more stable color in LL than PM. However, $a^{\star}$ values of Hp-LL increased $(\mathrm{p}<0.05)$, and the muscle remained dark, with very low $L^{\star}$ values, as mentioned previously, which is in general agreement with previous reports $[8,28,30]$, indicating a more stable color in LL than PM. Abril et al [31] demonstrated that $a^{*}$ values in Hp beef slightly increased and were greater than those in Np beef after day 6 of display, an observation not supported by the results of the current study.

The Hp-LL exhibited lower $(\mathrm{p}<0.05) a^{*}$ values than NpLL throughout display. Generally, $a^{\star}$ values were negatively correlated $(\mathrm{p}<0.05)$ with muscle $\mathrm{pH}(\mathrm{r}=-0.40$; Page, 2001). McKeith et al [13] and Apple et al [32] reported that redness in LL from Hp beef was lower than that in Np beef. However, in the present study, the $a^{\star}$ values of Hp-PM were not different from Np-PM, and the $\Delta \mathrm{a}^{*}$ was larger in $\mathrm{Hp}$ samples than $\mathrm{Np}$ beef during display. This indicates that the color stability of $\mathrm{Hp}-\mathrm{PM}$ is unaffected by the elevated $\mathrm{pH}$, or maybe the $\mathrm{pH}$ elevation was not large enough to promote further darkening, and thus, Hp-PM is still as labile as Np-PM. Although PM from both pHu groups showed a less stable color than LL from $\mathrm{Np}$ beef, the $a^{\star}$ values on day 7 were above the threshold (14.5) accepted by consumers [33].

The $b^{\star}$ values of Np-LL remained stable ( $\left.\mathrm{p}>0.05\right)$, while $b^{\star}$ values of PM of both normal and Hp groups decreased $(\mathrm{p}<0.05)$ during display. The differences in $b^{*}$ values of different muscles were similar to the differences in $a^{*}$ values. In support, Wu et al [34] reported a similar trend in both LL and PM from Np beef during storage. Joseph et al [7] reported that $b^{*}$ values were similar between LL and PM from Np beef during display except on day 5 .

Chroma value indicates the redness intensity. In the present study, the results for chroma values for both muscles during display were similar to the surface redness. The observed chroma values in LL and PM from Np beef throughout dis- 
play were in agreement with previous studies [7,30]. As for the effect of pHu, Apple et al [32] found greater chroma values in LL from Np beef than that in Hp beef, and increased chroma values in LL from Hp beef with extended display, consistent with the results reported here (Table 3 ).

Hue is a measure of color, with hue angle of 0 equal to red, and hue angle 90 equal to yellow (Minolta, Osaka, Japan; 1994). Both the interaction of muscle type $\times$ display time and the interaction of muscle type $\times \mathrm{pHu}$ had effects $(\mathrm{p}<0.05)$ on hue (Tables 3,4$)$. The hue values of both LL and PM increased $(\mathrm{p}<0.05)$ during display, indicative of decreased redness and increased yellowness during display. Np-PM had the highest $(\mathrm{p}<0.05)$ hue values, followed by Np-LL and Hp-PM exhibiting similar hue values, whereas Hp-LL had the lowest $(\mathrm{p}<0.05)$ hue values among muscles. In agreement, McKeith et al [13] reported that as Hp severity of LL increased, the hue values decreased. Kim et al [35] documented that hue in Np-PM increased, but remained stable in LL during display. According to the results (Table 3), we confirmed that LL of both $\mathrm{pHu}$ groups had greater red color stability than PM, emphasizing that the effect of muscle type needs to be considered when reporting on the effect of $\mathrm{pHu}$ on color stability during display.

R630/580: R630/580 was calculated to indicate meat surface color changes during storage or retail display, in which a greater ratio means a lesser amount of $\mathrm{MMb}$. As storage time increases, samples with a higher ratio have greater red color stability. There was an interaction $(\mathrm{p}<0.05)$ of $\mathrm{pHu} \times$ muscle type $\times$ display time for R630/580 (Table 3). R630/580 of NpLL was higher than other muscles and the values significantly decreased $(\mathrm{p}<0.05)$ in both muscles (Table 3$)$ over time. Although PM had greater R630/580 ( $<<0.05)$ than LL on the initial day, LL muscle showed greater R630/580 $(\mathrm{p}<0.05)$ than $\mathrm{PM}$ from day 3 to 7 , which was consistent with previous results $[7,8]$. The results for R630/580 in our study indicated a more rapid accumulation of $\mathrm{MMb}$ on the surface of PM steaks than on LL, reconfirming that PM is a color-labile muscle and $\mathrm{LL}$ is a color-stable muscle, regardless of $\mathrm{pHu}$.

Table 4. Effects of muscle type and display time on metmyoglobin reducing activity

\begin{tabular}{|c|c|c|c|c|c|c|}
\hline \multirow{2}{*}{ Trait } & \multirow{2}{*}{$\begin{array}{l}\text { Muscle } \\
\text { type }\end{array}$} & \multicolumn{4}{|c|}{ Display time (d) } & \multirow{2}{*}{ SE } \\
\hline & & 0 & 3 & 5 & 7 & \\
\hline \multirow[t]{2}{*}{ IMF $^{1)}$} & $\mathrm{LL}$ & $0.63^{\mathrm{ax}}$ & $0.66^{b x}$ & $0.64^{\mathrm{abx}}$ & $0.66^{\mathrm{abx}}$ & 0.01 \\
\hline & PM & $0.68^{\text {ay }}$ & $0.78^{\text {by }}$ & $0.78^{\text {by }}$ & $0.77^{\text {by }}$ & \\
\hline
\end{tabular}

MRA, metmyoglobin reducing activity; SE, standard error; IMF, initial metmyoglobin formation; LL, longissimus lumborum; PM, psoas major.

1) IMF was used to measure MRA and for which a lower value indicates greater MRA.

$a, b$ Means in the rows with different letters differ at $p<0.05$.

${ }^{x, y}$ Means in the columns with different letters differ at $p<0.05$.
Relative content of deoxymyoglobin, oxymyoglobin, and metmyoglobin

There was an interaction of $\mathrm{pHu} \times$ muscle type $\times$ display time $(\mathrm{p}<0.05)$ on $\% \mathrm{DMb}$ and $\% \mathrm{OMb}$. Moreover, both the interaction of muscle type $\times$ display time and the interaction of muscle type $\times \mathrm{pHu}$ had effects on steak surface $\% \mathrm{MMb}$ (Figure 1). During display, the \% DMb of Np-LL remained stable, whereas the $\% \mathrm{DMb}$ significantly decreased $(\mathrm{p}<0.05)$ from day 0 to day 3 in the other three muscles. For Hp-LL, $\% \mathrm{DMb}$ was greatest $(\mathrm{p}<0.05)$ at most time points and $\% \mathrm{DMb}$ at day 7 of display was less than half of the initial value. The results for \% OMb correspond to the color values, corroborating the results of $a^{*}$ and chroma values. An increase ( $\mathrm{p}<$ $0.05)$ of $\% \mathrm{MMb}$ in both LL and PM was observed, and as expected, the \% MMb was higher $(\mathrm{p}<0.05)$ in the PM than in the LL at all display days. Previous study reported that $\mathrm{Np}-\mathrm{PM}$ had faster and greater \% MMb accumulation and lower \% OMb than Np-LL during display [5]. English et al [12] documented that Hp beef had greater \% DMb than Np beef, due to high $\mathrm{pH}$ values which enhances mitochondrial respiration, leaving less oxygen available to bind to surface myoglobin, leading to more $\mathrm{DMb}$ formation [12]. The similar $\% \mathrm{OMb}$ from $\mathrm{PM}$ between $\mathrm{Np}$ and $\mathrm{Hp}$ beef, resulted in the similar redness of these samples during display. However, the significantly decreased redness of Hp-PM during display was the result of the accumulation of $\mathrm{MMb}$ that was

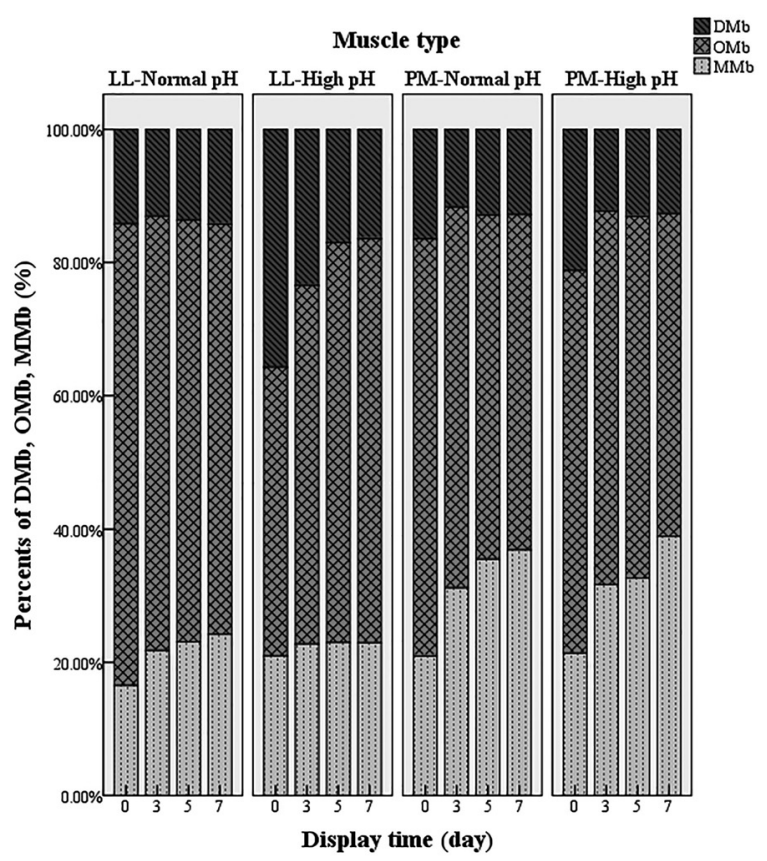

Figure 1. Effects of display time, muscle type (Longissmus lumborum [LL] vs psoas major [PM]) and ultimate $\mathrm{pH}$ (normal pH vs dark cutting) on relative content of three myoglobin forms (deoxymyoglobin, DMb; oxymyoglobin, OMb, and metmyoglobin, MMb). The content of $\mathrm{DMb}, \mathrm{OMb}$, and $\mathrm{MMb}$ was calculated by meat color measurement guidelines established by American Meat Science Association. 
as high as in NP-PM.

\section{Metmyoglobin reducing activity}

There were interactions between muscle type $\times$ display $(\mathrm{p}<$ $0.05)$ and muscle type $\times \mathrm{pHu}(\mathrm{p}<0.05)$ for initial metmyoglobin formation (IMF) (defined as initial MMb formation, high value indicates lower MRA; Tables 4, 5). For both LL and PM, IMF increased $(\mathrm{p}<0.05)$ during the first 3 days of display, with the extent more pronounced $(\mathrm{p}<0.05)$ in the $\mathrm{PM}$, which also exhibited higher $(\mathrm{p}<0.05)$ value throughout display, indicating that the PM had a lower capacity to reduce $\mathrm{MMb}$ and poorer color stability. Furthermore, IMF and $\Delta \%$ $\mathrm{MMb}$ were positively correlated $(\mathrm{r}=0.96, \mathrm{p}<0.05$, data not shown). Hp-LL had lowest IMF and the IMF in the Np-PM and Hp-PM were similar (Table 5). Noticeably, IMF in NpLL was lower than that in the Hp-PM, which is consistent with the results for R630/580 and relative MMb content, reinforcing that the color stability was greater in Np-LL than in the Hp-PM.

Previous studies in Np muscles demonstrated that MRA decreased in LL and PM muscles across display and LL exhibited greater MRA than PM, which is in agreement with our results [7,34]. Similarly, Ke et al [36] reported that LL had greater MRA than PM, and MRA in PM drastically decreased during the first three days of display. In support, English et al [12] documented that Hp-LL had greater MRA than NpLL during ageing. The decreased MRA during display and differences between muscle types could be explained by substrate depletion, nicotinamide-adenine dinucleotide (NADH) regeneration and the activity of NADH- cytochrome b5 reductase, which is necessary for both enzymatic and nonenzymatic reducing pathways [37].

\section{Oxygen consumption}

There was a significant interaction effect of muscle type $\times \mathrm{pHu}$ (OC; Table 5). Compared with Hp-LL and Np-PM, Np-LL showed lower OC $(\mathrm{p}<0.05)$, while remarkably, the PM of Hp beef had higher $(\mathrm{p}<0.05)$ OC than the LL of Np beef. Previous studies have demonstrated that OC decreased in muscle with increasing days of display $[5,36]$. The decreased OC could be explained by the reduction in the functional ability of the mitochondria due to extended post-mortem time and depleted substrates [37]. In support, Hp beef exhibited greater mitochondrial activity and OC than Np beef, as reported previously [12]. In general, PM exhibited greater mitochondrial content and OC than LL of Np beef, as reported by Mancini et al [30]. The increased mitochondrial activity and content are responsible for the increased OC.

High OC enhances the capacity of mitochondria to compete for available oxygen with myoglobin, resulting in more $\mathrm{DMb}$ or MMb formation $[5,6]$. It has been reported that $\mathrm{OC}$ plays a more crucial role in color stability, compared with MRA. Seyfert et al [6] found that if the level of MRA in the muscle could not match the oxidative stress resulting from OC, it would exhibit poorer color stability. The results presented here indicates that Np-LL, which had a similar OC to Hp-PM, showed greater color stability, all because of the much greater MRA of Np-LL than Hp-PM. But PM of both $\mathrm{pHu}$ groups had almost identical MRA and OC, exhibiting similar color stability.

\section{Lipid oxidation}

The interaction of muscle type and $\mathrm{pHu}$ was significant for thiobarbituric acid reactive substances (TBARS) values (Table 5). Hp-LL had lower TBARS values than the other three muscles from day 3 , while the significant difference was only found on day 7 , and the remaining three samples did not differ ( $p>0.05)$. Hp-LL had much lower $(\mathrm{p}<0.05)$ fat content than Np LL (0.84\% vs $1.96 \%$, respectively; Table 1$)$, and correspondingly lower TBARS values ( 0.22 vs 0.33 ; Table 5$)$. The higher fat content and TBARS of the Np-LL indicates that lipid oxidation products were possibly contributing to the loss of redness observed in Np-LL at 7 days display, compared to Hp-LL.

In support of our results, Sawyer et al [27] found that LL from $\mathrm{Np}$ and $\mathrm{Hp}$ beef showed different TBARS values during the first 5-days of display, however, without a significant difference. Likewise, English et al [12] reported greater TBARS values in Np-LL than in $\mathrm{Hp}$ beef under vacuum packaging. Purohit et al [38] found a strong and negative correlation $(-0.93)$ in LL between TBARS values and $\mathrm{pH}$, supporting the observed results in the LL.

Table 5. Interaction effects of muscle type and ultimate pH on MRA, OC, and TBARS values

\begin{tabular}{|c|c|c|c|c|c|}
\hline \multirow{2}{*}{ Trait } & \multicolumn{2}{|c|}{ LL } & \multicolumn{2}{|c|}{ PM } & \multirow{2}{*}{ SE } \\
\hline & Normal pH & High pH & Normal pH & High pH & \\
\hline IMF $^{1)}$ & $0.71^{\mathrm{c}}$ & $0.59^{d}$ & $0.74^{b}$ & $0.77^{\mathrm{a}}$ & 0.01 \\
\hline OC & $0.56^{c}$ & $0.64^{\mathrm{a}}$ & $0.63^{\mathrm{a}}$ & $0.59^{b}$ & 0.01 \\
\hline TBARS (malondialdehhyde mg/kg) & $0.33^{\mathrm{a}}$ & $0.22^{b}$ & $0.31^{a b}$ & $0.36^{\mathrm{a}}$ & 0.06 \\
\hline
\end{tabular}

MRA, metmyoglobin reducing activity; OC, oxygen consumption; TBARS, thiobarbituric acid reactive substances; LL, longissimus lumborum; PM, psoas major; SE, standard error; IMF, initial metmyoglobin formation.

1) IMF was used to measure MRA and for which a lower value indicates greater MRA.

${ }^{a-d}$ Means in the rows with different letters differ at $p<0.05$. 
Similar to our findings, Jeong et al [39] documented that there was no difference in TBARS values between Longissimus and PM from Np beef throughout display periods, although both muscles had different fat contents. Partially in agreement with our results, several previous investigations in $\mathrm{Np}$ muscle reported that TBARS values increased in LL and PM over time. However, greater TBARS values were observed in PM than LL $[8,36]$. Generally, color-stable muscle (as LL) or/and LL muscle with high $\mathrm{pH}$ showed lower TBARS. Noticeably, lipid oxidation between Np-LL (color-stable muscle) and Hp-PM (high pH muscle) were not different. The reason for this is not clear.

Taken together, LL exhibited greater color stability than the $\mathrm{PM}$ when the $\mathrm{pH}$ is normal, which is attributed to the higher $\mathrm{MMb}$ accumulation in $\mathrm{PM}$, resulting from its higher OC and lower MRA than the LL. High $\mathrm{pH}$ also resulted in a greater color stability, i.e. dark cutting LL had greatest color stability among all beef samples. PM from $\mathrm{Hp}$ beef exhibited lower color stability than LL from Np beef; this is also caused by the high accumulation of MMb, lower MRA and greater $\mathrm{OC}$ in the former sample. Thus, the order of color stability is LL of Hp beef, LL of Np beef, followed by PM of both $\mathrm{pHu}$ groups.

\section{CONCLUSION}

Hp beef does not always show better color stability compared with Np beef, which depends on the muscle type. The balance of MRA and OC is important to keep the color in great intensity and stability in the meantime. Additionally, although redness intensity in Hp-LL was improved through display, we must consider microbiological safety and note that the TVC in Hp reached above $6.0 \log \mathrm{CFU} / \mathrm{g}$ at 7-days display. These findings should aid the processing and retailing of LL and PM from different $\mathrm{pHu}$ beef.

\section{CONFLICT OF INTEREST}

We certify that there is no conflict of interest with any financial organization regarding the material discussed in the manuscript.

\section{ACKNOWLEDGMENTS}

This work was supported by the projects of National Natural Science Fund (31601528 and 31871731), the National Beef Cattle Industrial Technology System (CARS-37), and special fund for innovation team of modern agricultural industrial technology system in Shandong Province (SDAIT-09-09).

\section{REFERENCES}

1. Mancini RA, Hunt MC. Current research in meat color. Meat Sci 2005;71:100-21. https://doi.org/10.1016/j.meatsci.2005. 03.003

2. Suman SP, Joseph P. Myoglobin chemistry and meat color. Annu Rev Food Sci Technol 2013;4:79-99. https://oi.org/ 10.1146/annurev-food-030212-182623

3. Smith GC, Belk KE, Sofos JN, Tatum JD, Williams SN. Economic implications of improved color stability in beef. In: Decker E, Faustman C, Lopez-Bote CJ editors. Antioxidants in muscle foods: nutritional strategies to improve quality. New York, USA: Wiley Interscience; 2000. pp. 397-426.

4. McKenna DR, Roebert DL, Bates PK, et al. National Beef Quality Audit-2000: survey of targeted cattle and carcass characteristics related to quality, quantity, and value of fed steers and heifers. J Anim Sci 2002;80:1212-22. https://doi. org/10.2527/2002.8051212x

5. McKenna DR, Mies PD, Baird BE, Pfeiffer KD, Ellebracht JW, Savell JW. Biochemical and physical factors affecting discoloration characteristics of 19 bovine muscles. Meat Sci 2005;70:665-82. https://doi.org/10.1016/j.meatsci.2005.02.016

6. Seyfert M, Mancini RA, Hunt MC, Tang J, Faustman C, Garcia M. Color stability, reducing activity, and cytochrome c oxidase activity of five bovine muscles. J Agric Food Chem 2006;54: 8919-25. https://doi.org/10.1021/jf061657s

7. Joseph P, Suman SP, Rentfrow G, Li S, Beach CM. Proteomics of muscle-specific beef color stability. J Agric Food Chem 2012;60:3196-203. https://doi.org/10.1021/jf204188v

8. Canto ACVCS, Costa-Lima BRC, Suman SP, et al. Color attributes and oxidative stability of longissimus lumborum and psoas major muscles from Nellore bulls. Meat Sci 2016; 121:19-26. https://doi.org/10.1016/j.meatsci.2016.05.015

9. Ashmore CR, Doerr L, Foster G, Carroll F. Respiration of mitochondria isolated from dark-cutting beef. J Anim Sci 1971;33:574-7. https://doi.org/10.2527/jas1971.333574x

10. Ponnampalam EN, Hopkins DL, Bruce H, Li D, Baldi G, Bekhit AE. Causes and contributing factors to "dark cutting" meat: current trends and future directions: a review. Compr Rev Food Sci Food Saf 2017;16:400-30. https://doi.org/10.1111/ 1541-4337.12258

11. Hughes J, Clarke F, Purslow P, Warner R. High pH in beef longissimus thoracis reduces muscle fibre transverse shrinkage and light scattering which contributes to the dark colour. Food Res Int 2017;101:228-38. https://doi.org/10.1016/j.foodres. 2017.09.003

12.English AR, Wills KM, Harsh BN, Mafi GG, Vanoverbeke DL, Ramanathan R. Effects of aging on the fundamental color chemistry of dark-cutting beef. J Anim Sci 2016;94:40408. https://doi.org/10.2527/jas.2016-0561

13. McKeith RO, King DA, Grayson AL, et al. Mitochondrial abundance and efficiency contribute to lean color of dark cutting beef. Meat Sci 2016;116:165-73. https://doi.org/10. 1016/j.meatsci.2016.01.016 
14. Wulf DM, Emnett RS, Leheska JM, Moeller SJ. Relationships among glycolytic potential, dark cutting (dark, firm, and dry) beef, and cooked beef palatability. J Anim Sci 2002;80:1895903. https://doi.org/10.2527/2002.8071895x

15. Holman BWB, Hopkins DL. Contrasting the quality traits of aged bolar blade, topside and striploin cuts sourced from dark cutting and control Australian beef carcasses. Meat Sci 2019;149:24-30. https://doi.org/10.1016/j.meatsci.2018.10.014

16. Du Y, Zhang J, Hu T, Luo X. Effect of pre-slaughter conditions on beef quality. Scientia Agricultura Sinica 2009;42:3625-32. (In Chinese, with English abstract). https://doi.org/10.3864/j. issn.0578-1752.2009.10.0030

17.AMSA. Meat color measurement guidelines. Champaign, IL, USA: American Meat Science Association; 2012.

18. Mancini RA, Seyfert M, Hunt MC. Effects of data expression, sample location, and oxygen partial pressure on initial nitric oxide metmyoglobin formation and metmyoglobin-reducingactivity measurement in beef muscle. Meat Sci 2008;79:24451. https://doi.org/10.1016/j.meatsci.2007.09.008

19. Sammel LM, Hunt MC, Kropf DH, Hachmeister KA, Johnson DE. Comparison of assays for metmyoglobin reducing ability in beef inside and outside semimembranosus muscle. J Food Sci 2002;67:978-84. https://doi.org/10.1111/j.1365-2621.2002. tb09439.x

20. Madhavi DL, Carpenter CE. Aging and processing affect color, metmyoglobin reductase and oxygen consumption of beef muscles. J Food Sci 1993;58:939-42. https://doi.org/10.1111/ j.1365-2621.1993.tb06083.x

21.Siu GM, Draper HH. A survey of the malonaldehyde content of retail meats and fish. J Food Sci 1978;43:1147-9. https:// doi.org/10.1111/j.1365-2621.1978.tb15256.x

22. Yu Q, Wu W, Tian X, et al. Comparative proteomics to reveal muscle-specific beef color stability of Holstein cattle during post-mortem storage. Food Chem 2017;229:769-78. https:// doi.org/10.1016/j.foodchem.2017.03.004

23. Lefaucheur L. A second look into fibre typing--relation to meat quality. Meat Sci 2010;84:257-70. https://doi.org/10. 1016/j.meatsci.2009.05.004

24. England EM, Matarneh SK, Oliver EM, et al. Excess glycogen does not resolve high ultimate $\mathrm{pH}$ of oxidative muscle. Meat Sci 2016;114:95-102. http://doi.org/10.1016/j.meatsci.2015. 10.010

25. Gardner GE, Hopkins DL, Greenwood PL, Cake MA, Boyce MD, Pethick DW. Sheep genotype, age and muscle type affect the expression of metabolic enzyme markers. Aust J Exp Agric 2007;47:1180-9. https://doi.org/10.1071/EA07093

26. Rule DC. Lipids in muscle: struture, composition and metabolism. In: Du M, McCormick R, editors. Applied muscle biology and meat science. Boca Raton, FL, USA: Taylor \& Francis Group; 2009. pp. 47-74.

27.Sawyer JT, Apple JK, Johnson ZB, Baublits RT, Yancey JWS. Fresh and cooked color of dark-cutting beef can be altered by post-rigor enhancement with lactic acid. Meat Sci 2009;83: 263-70. https://doi.org/10.1016/j.meatsci.2009.05.008

28. Ma D, Kim YHB, Cooper B, et al. Metabolomics profiling to determine the effect of postmortem aging on color and lipid oxidative stabilities of different bovine muscles. J Agric Food Chem 2017;65:6708-16. https://doi.org/10.1021/acs.jafc. $7 \mathrm{~b} 02175$

29. Mahmood S, Turchinsky N, Paradis F, Dixon WT, Bruce HL. Proteomics of dark cutting longissimus thoracis muscle from heifer and steer carcasses. Meat Sci 2018;137:47-57. https:// doi.org/10.1016/j.meatsci.2017.11.014

30. Mancini RA, Belskie K, Suman SP, Ramanathan R. Musclespecific mitochondrial functionality and its influence on fresh beef color stability. J Food Sci 2018;83:2077-82. https:// doi.org/10.1111/1750-3841.14219

31. Abril M, Campo MM, Onenç A, C Sañudo, P Albertí, Negueruela AI. Beef colour evolution as a function of ultimate $\mathrm{pH}$. Meat Sci 2001;58:69-78. https://doi.org/10.1016/S0309-1740 (00)00133-9

32. Apple JK, Sawyer JT, Meullenet JF, Yancey JWS, Wharton MD. Lactic acid enhancement can improve the fresh and cooked color of dark-cutting beef. J Anim Sci 2011;89:4207-20. https://doi.org/10.2527/jas.2011-4147

33. Holman BWB, van de Ven RJ, Mao Y, Coombs CEO, Hopkins DL. Using instrumental (CIE and reflectance) measures to predict consumers' acceptance of beef colour. Meat Sci 2017; 127:57-62. https://doi.org/10.1016/j.meatsci.2017.01.005

34. Wu W, Yu Q, Fu Y, et al. Towards muscle-specific meat color stability of Chinese Luxi yellow cattle: A proteomic insight into post-mortem storage. J Proteomics 2016;147:108-18. https://doi.org/10.1016/j.jprot.2015.10.027

35. Kim YH, Keeton JT, Smith SB, Berghman LR, Savell JW. Role of lactate dehydrogenase in metmyoglobin reduction and color stability of different bovine muscles. Meat Sci 2009; 83:376-82. http://doi.org/10.1016/j.meatsci.2009.06.009

36. Ke Y, Mitacek RM, Abraham A, et al. Effects of muscle-specific oxidative stress on cytochrome $c$ release and oxidation-reduction potential properties. J Agric Food Chem 2017;65:774955. https://doi.org/10.1021/acs.jafc.7b01735

37. Tang J, Faustman C, Mancini RA, Seyfert M, Hunt MC. Mitochondrial reduction of metmyoglobin:? dependence on the electron transport chain. J Agric Food Chem 2005;53:544955. https://doi.org/10.1021/jf050092h

38. Purohit A, Singh R, Kerr W, Mohan A. Effects of heme and nonheme iron on meat quality characteristics during retail display and storage. J Food Meas Charact 2015;9:175-85. https://doi.org/10.1007/s11694-015-9222-y

39. Jeong JY, Hur SJ, Yang HS, et al. Discoloration characteristics of 3 major muscles from cattle during cold storage. J Food Sci 2009;74:C1-5. https://doi.org/10.1111/j.1750-3841.2008. 00983.x 